This is the peer reviewed version of the paper:

Denysenko I. B., Stefanović Ilija, Mikikian M., Kovačević E., Berndt J., "Argon/dust and pure argon pulsed plasmas explored using a spatially-averaged model" Journal of Physics D: Applied Physics, 54, no. 6 (2021):065202, https://doi.org/10.1088/1361$\underline{6463 / \mathrm{abc} 210}$

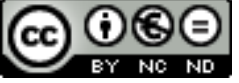

This work is licensed under a Creative Commons Attribution Non Commercial No Derivatives 4.0 license 


\title{
Argon/dust and pure argon pulsed plasmas explored using a spatially-averaged model
}

\author{
I B Denysenko ${ }^{1,2,3}$, I Stefanović ${ }^{4}$, M Mikikian' ${ }^{2}$ E Kovacevic ${ }^{2}$ and J Berndt ${ }^{2}$ \\ ${ }^{1}$ School of Physics and Technology, V N Karazin Kharkiv National University, Kharkiv, Ukraine \\ ${ }^{2}$ GREMI, UMR7344 CNRS/Université d'Orléans, F-45067 Orléans, France \\ ${ }^{3}$ Le Studium, Loire Valley Institute for Advanced Studies, Orléans \& Tours, France \\ ${ }^{4}$ Institute of Technical Sciences, Serbian Academy of Sciences \& Arts, 11000, Belgrade, Serbia
}

E-mail: idenysenko@yahoo.com

Received

Accepted for publication

Published

\begin{abstract}
The properties (densities of electrons and metastable argon atoms, effective electron temperature and dust charge) of argon/dust and pure argon pulsed plasmas are studied using a spatially-averaged model. The calculated time-dependencies for the densities of electrons and metastable atoms are compared with the experimental measurements and are found to be in a good qualitative agreement. It is analyzed how the plasma properties depend on the shape of the electron energy probability function (EEPF), the pulsing frequency and the duty cycle for both dust-free and dusty plasma. The analysis reveals that the agreement between theory and experiment is better with Druyvesteyn EEPF than the Maxwellian EEPF. Further, the variation in the pulsing frequency $v_{\mathrm{p}}$ differently affects the metastable density $n_{\mathrm{m}}$ in a dust-free and in a dusty plasma. For large $v_{\mathrm{p}}$, the metastable density in the dust-free pulsed plasma is larger than in the continuous-wave (CW) discharge, while the opposite is obtained in the presence of dust particles. This difference probably arises because of faster variation in the effective electron temperature in the dusty plasma due to collection of electrons by dust particles. Our calculations also show that dust particles may affect the behavior of electron density in the beginning of the on-period due to an enhancement in electron collection by dust particles.
\end{abstract}

Keywords: plasma, dust particles, pulsing frequency, duty cycle, spatially-averaged model

\section{Introduction}

Gas discharges containing charged nano and micro particles (dust particles) have been extensively studied for several decades $[1-3]$ as these dusty plasmas are involved in many technological and bioimaging applications and also in fusion research $[1,4-6]$. They are also of a fundamental interest and have attracted research attention in diverse fields, including the formation of Coulomb dust crystals, dust vortices and voids, cosmic clouds, etc. [1-3, 7, 8]

Many properties of gas discharges with dust particles have been investigated both experimentally and numerically focusing mostly to the continuous wave $(\mathrm{CW})$ regime $[1-3,7-$ 13]. However, in many technological applications, plasma systems are not stationary [14]. Nonstationary gas discharges (especially pulsed systems) are often employed for reduction of charge accumulation on the substrates, improving the quality of the deposited films and etch selectivity [14, 15], controlling particle formation in chemically active plasmas, etc. [16-18] Therefore, dust particles in nonstationary gas discharges and afterglow plasmas have also been studied by some authors [13, 19-21].

In [19], the time-dependence of the charge of micrometer dust particles in the afterglow of a RF plasma under microgravity conditions was analyzed experimentally and theoretically. It was found that dust particles keep a negative charge for a long time in the afterglow. According to [20], negatively and positively charged dust particles as well as neutral ones coexisted for more than a minute in a RF discharge afterglow. By analyzing the dust particle diffusion in the afterglow, several properties of a dusty plasma are established [21]. 
A symmetrically - driven RF discharge was used to analyze the pulsed dusty plasmas with large dust charge densities $\left(\left|n_{d} Z_{d}\right| \geq n_{e}\right.$, where $Z_{d}$ is the dust charge number in units of elementary charge $e, n_{e}$ and $n_{d}$ are the densities of electrons and dust particles, respectively) [22-25]. Dust particles were first formed in $\mathrm{Ar} / \mathrm{C}_{2} \mathrm{H}_{2}$ plasma and then the acetylene input was stopped to keep only an argon/dusty plasma. However, acetylene could remain in the chamber from the growth phase or due to the desorption of $\mathrm{C}_{2} \mathrm{H}_{2}$ from the chamber walls. The experiments showed that the electron density increased unexpectedly at the very beginning of the dusty plasma afterglow [24, 25]. Several mechanisms were proposed to describe this phenomenon. Firstly, the increase of the electron density in the early afterglow was attributed to the release of electrons from the dust particles by secondary electron emission in ion-dust collisions [25]. The other sources of electrons in the early afterglow were identified and compared, such as electron generation in metastablemetastable and metastable-dust collisions [22] or secondary electron emission from electrodes [26]. In a presence of impurities, such as acetylene, the metastable - acetylene collisions make a significant contribution to the described phenomenon [27].

Note that in most theoretical studies of nonstationary dusty plasmas, the electron energy probability function (EEPF) was assumed to be Maxwellian, whereas, in practice, the EEPF in low-temperature plasmas is usually nonMaxwellian [28-30]. Moreover, in [22-26] the authors focused their attention mainly on the off-period (afterglow). Therefore, these studies did not provide information about the time variation of plasma properties during the on-period and about the dependence of plasma properties on the pulsing parameters (frequency $v_{\mathrm{p}}$, duty cycle $\eta$ ). Nevertheless, this information is important to control pulsed plasma properties in different technological applications [14, 16, 31].

In this paper, we propose a theoretical description for a pulsed dusty plasma with large dust charge densities. The current model formulation is based on a spatially-averaged (0D) model, which describes both the on- and off-periods of the pulsed plasma and accounts for the non-Maxwellian shape of the EEPF. Thus, this 0D model differs significantly from the models used in our previous studies of pulsed dusty plasmas, where only the afterglow is considered and the electrons are assumed to be Maxwellian [22, 26]. Beside of that, the model uses recently proposed rate constant for argon metastable - metastable collisions (known also as "metastable pooling"), and the new Penning branching ratio in $\mathrm{Ar}^{*}-\mathrm{C}_{2} \mathrm{H}_{2}$ reaction $\alpha=0.3$ [27]. The metastable pooling rate is a few times lower compared to the value commonly used in the literature [32]. The model is used to study plasma properties (the electron and metastable atoms' densities, effective electron temperature and dust charge) as a function of time in pure argon and dusty pulsed discharges. It is also studied how the plasma properties depend on the shape of the EEPF, the pulsing frequency and the duty cycle. For the sake of comparison, the studies are carried out for the conditions close to those in the experiments $[16,25,27]$. The calculated timedependencies for densities of electrons and metastable argon atoms are compared with measurements from $[22,27]$ for dust-free and dusty plasma.

\section{Theoretical model}

In the $0 \mathrm{D}$ model, it is assumed that the gas-discharge plasma has $R=15 \mathrm{~cm}$ radius and $L=7 \mathrm{~cm}$ height and consists of electrons with density $n_{\mathrm{e}}$, singly charged positive ions $\left(\mathrm{Ar}^{+}\right)$ with density $n_{\mathrm{i}}$, dust particles with density $n_{\mathrm{d}}$, radius $a_{\mathrm{d}}$ and negative charge $e \mathrm{Z}_{\mathrm{d}}$, ground-state argon atoms $\left(\mathrm{Ar}_{0}\right)$ with density $n_{\mathrm{a}}$, metastable argon atoms $\left(\mathrm{Ar}_{\mathrm{m}}\right)$ with density $n_{\mathrm{m}}$, argon atoms in the resonance $4 \mathrm{~s}$ states $\left({ }^{3} \mathrm{P}_{1}\right.$ and $\left.{ }^{1} \mathrm{P}_{1}\right)\left(\mathrm{Ar}_{\mathrm{r}}\right)$ with density $n_{\mathrm{r}}$ as well as argon atoms in $4 \mathrm{p}$ states $\left(\mathrm{Ar}_{4 \mathrm{p}}\right)$ with density $n_{4 \mathrm{p}}$. The metastable and resonance atom densities $n_{\mathrm{m}}$, $n_{\mathrm{r}}$ and $n_{4 \mathrm{p}}$ represent the density of a composite $\left({ }^{3} \mathrm{P}_{0}\right.$ and $\left.{ }^{3} \mathrm{P}_{2}\right)$ metastable level, the density of a composite $\left({ }^{3} \mathrm{P}_{1}\right.$ and $\left.{ }^{1} \mathrm{P}_{1}\right)$ resonance level and the density of a composite $4 p$ state, respectively. It is assumed that ions and dust particles are at gas temperature $T_{\mathrm{g}}$ and ions have Maxwellian distribution. In numerical simulations, the gas temperature $T_{\mathrm{g}}$ is considered to be $294 \mathrm{~K}$ and $366 \mathrm{~K}$ in the dust-free and dusty cases, respectively [33].

The electron energy probability function (EEPF) is described by the following expression [34]

$$
F(\varepsilon)=A_{1} \exp \left(-A_{2} \varepsilon^{x}\right) \text {, }
$$

where $\varepsilon$ is the electron energy and $x$ takes different values according to the shape of EEPF: $x=1$ and $x=2$ for Maxwellian and Druyvesteyn electron energy distributions, respectively. The coefficients $A_{1}$ and $A_{2}$ are functions of $x$ and the average electron energy [34] $\langle\varepsilon\rangle=\int_{0}^{\infty} \varepsilon F(\varepsilon) \sqrt{\varepsilon} d \varepsilon=$ $\frac{3}{2} e T_{\text {eff }}$ and $T_{\text {eff }}$ is the effective electron temperature. The EEPF has the following normalization $\int_{0}^{\infty} F(\varepsilon) \sqrt{\varepsilon} d \varepsilon=1$.

In most of the cases considered in the next section, we will assume that the EEPF is Druyvesteyn-like. This distribution is typical for laboratory plasmas sustained by an RF generator with the frequency $f=13.56 \mathrm{MHz}$ at $n_{e}<10^{11}$ $\mathrm{cm}^{-3}$ and $P d>0.2$ Torr $\times \mathrm{cm}$ [35], where $P$ is the argon gas pressure and $d \approx L / 2$ is the dimension of the reactor in $\mathrm{cm}$. However, effects of variation of the EEPF shape on plasma properties will be also studied.

The particle balance equation for a species $X$ (electrons and argon atoms in excited states) is given as

$$
\frac{\partial n^{(X)}}{\partial t}=\sum_{i} R_{G, i}^{(X)}-\sum_{i} R_{L, i}^{(X)},
$$

where $R_{G, i}^{(X)}$ and $R_{L, i}^{(X)}$ are, respectively, the rates for reactions of the various generation and loss processes of the species $X$. It is assumed that in both pure argon and dusty pulsed plasmas, electrons are generated in collisions of electrons with argon atoms in the ground and excited states ( $4 \mathrm{p}$ and $4 \mathrm{~s}$ states), as well as in metastable-metastable collisions (metastable pooling). In the dusty plasma, we assume that electrons can be additionally produced in one of the two processes: secondary 
electron emission in collisions of excited argon atoms $\operatorname{Ar}^{*}\left(\operatorname{Ar}_{\mathrm{m}}, \mathrm{Ar}_{\mathrm{r}}\right.$ and $\left.A \mathrm{Ar}_{4 \mathrm{p}}\right)$ with dust particles with the effective secondary emission yield $\gamma_{\mathrm{m}}$ [22] and interaction of excited atoms with acetylene molecules [27]. In the latter case, it is assumed that acetylene molecules are present in small amounts in the pulsed dusty plasma. The experimental procedure of making the dust plasma involves the use of acetylene as a precursor that could remain in the chamber from the previous phase of the experiment when the dust particles are formed in $\mathrm{Ar} / \mathrm{C}_{2} \mathrm{H}_{2}$ plasma, or due to the desorption of $\mathrm{C}_{2} \mathrm{H}_{2}$ from the chamber walls [27]. The interaction of excited atoms with acetylene molecules is characterized by the quenching rate coefficient $k_{\mathrm{q}}=5.6 \times 10^{-10} \mathrm{~cm}^{3} \mathrm{~s}^{-1}$ and by the Penning branching ratio in $\mathrm{Ar}^{*}-\mathrm{C}_{2} \mathrm{H}_{2}$ reaction $\alpha=0.3$ [27]. Here, we consider the acetylene as an impurity with the very low density $\left(\sim 10^{11} \mathrm{~cm}^{-3}\right)$ compared to the argon $\left(\sim 10^{15} \mathrm{~cm}^{-3}\right)$ in the dusty pulsed plasma. Therefore, these impurity molecules do not affect essentially the ion composition and the energy balance in the plasma volume.

The model assumes that electrons and ions are lost from the discharge because of their diffusion to the walls and by deposition on dust particles.

In the model, the ion density $n_{i}$ is found from the quasineutrality condition

$$
n_{i}-n_{e}+n_{d} Z_{d}=0 \text {. }
$$

Considering various collisional processes in the plasma volume, we account only for binary collisions. A reaction rate for such reactions is calculated as the product of the reactants' densities and the rate coefficient $K$ of the reaction, $R=K \times$ $n_{\alpha} n_{\beta}$, where $n_{\alpha}$ and $n_{\beta}$ are the densities of the reactants. The electron production and loss processes are listed in the table 1.

The rate coefficients for reactions (R1)-(R4) are calculated using the corresponding reaction cross sections $\sigma$ taken from [36-38] $\left(K=\int_{0}^{\infty} \sigma(\varepsilon) \sqrt{2 e \varepsilon / m_{e}} F(\varepsilon) \sqrt{\varepsilon} d \varepsilon\right.$, where $m_{\mathrm{e}}$ is the electron mass). In the most cases considered in our paper, the rate for metastable pooling is taken to be $K_{\mathrm{p}}=1.24$ $\times 10^{-10} \mathrm{~cm}^{3} / \mathrm{s}$ (about 10 times smaller than in [39], as it was proposed in [27]). The calculations are also carried out for the value $K_{\mathrm{p}}=6.2 \times 10^{-10} \mathrm{~cm}^{3} / \mathrm{s}$, which is commonly used in simulation works $[22,40]$.

The rate for electron diffusion to the walls $R_{\mathrm{w}}$ is calculated assuming that the total ion and electron fluxes to the walls are equal, i.e. $R_{W}=n_{i} k_{w i}$, where $k_{w i}=2 u_{B} \frac{R^{2} h_{L}+R L h_{R}}{R^{2} L}$. Here, $u_{B}=\langle\varepsilon\rangle^{1 / 2}\left(\frac{2 e}{m_{i}}\right)^{1 / 2} \frac{\Gamma\left(\xi_{1}\right)}{\left[\Gamma\left(\xi_{2}\right) \Gamma\left(\xi_{3}\right)\right]^{1 / 2}}$ is the Bohm velocity [34], $\Gamma(\xi)$ is the gamma function with $\xi_{1}=3 / 2 x, \xi_{2}=5 / 2 x$ and $\xi_{3}=1 / 2 x, m_{i}$ is the ion mass and $h_{L}$ and $h_{R}$ are the edge to centre positive ion density ratios [34]. The rate coefficient for collisions of argon atoms in excited states with dust particles is $K_{m}^{d}=\pi a_{d}^{2} \sqrt{8 e T_{i} / \pi m_{i}}$. The rate coefficient for deposition of electrons on negatively charged dust particles is calculated as $K_{e}^{d}=\pi a_{d}^{2} \int_{-\varphi_{s}}^{\infty}\left(1+\frac{\varphi_{s}}{\varepsilon}\right) \sqrt{\frac{2 e \varepsilon}{m_{e}}} F(\varepsilon) \sqrt{\varepsilon} d \varepsilon$, where $\varphi_{s}=\frac{e Z_{d}}{a_{d}}$ is the dust surface potential. In the case when acetylene impurity is in the plasma, we assume that $\mathrm{Ar}^{*}-\mathrm{C}_{2} \mathrm{H}_{2}$ collisions are accompanied by production of electrons (table 1) and by loss of $\mathrm{Ar}^{*}$ atoms (table 2). Since the density of $\mathrm{C}_{2} \mathrm{H}_{2}$ molecules is assumed to be very low here, we do not consider in our model the following products of this reaction: $\mathrm{C}_{2} \mathrm{H}_{2}{ }^{+}$, $\mathrm{C}_{2} \mathrm{H}$ and $\mathrm{H}$.

Table 1. Production and loss processes of electrons relevant for the model.

\begin{tabular}{|c|c|c|}
\hline Reaction & Rate & Reference \\
\hline$(\mathrm{R} 1) \mathrm{e}+\mathrm{Ar}_{0} \rightarrow \mathrm{Ar}^{+}+2 \mathrm{e}$ & $K^{R l}=f(F, \sigma)$ & [36] \\
\hline (R2) $\mathrm{e}+\mathrm{Ar}_{\mathrm{m}} \rightarrow \mathrm{Ar}^{+}+2 \mathrm{e}$ & $K^{R 2}=f(F, \sigma)$ & [37] \\
\hline$(\mathrm{R} 3) \mathrm{e}+\mathrm{Ar}_{\mathrm{r}} \rightarrow \mathrm{Ar}^{+}+2 \mathrm{e}$ & $K^{R 3}=f(F, \sigma)$ & [37] \\
\hline$(\mathrm{R} 4) \mathrm{e}+\operatorname{Ar}(4 \mathrm{p}) \rightarrow \mathrm{Ar}^{+}+2 \mathrm{e}$ & $K^{R 4}=f(F, \sigma)$ & [38] \\
\hline (R5) $\mathrm{Ar}_{\mathrm{m}}+\mathrm{Ar}_{\mathrm{m}} \underset{\mathrm{Ar}}{\rightarrow}+\mathrm{Ar}^{+}+\mathrm{e}$ & $K_{\mathrm{p}}$ & {$[27,40]$} \\
\hline (R6) $\mathrm{Ar}_{\mathrm{m}}+\mathrm{Ar}_{\mathrm{r}} \underset{\mathrm{Ar}}{\rightarrow}+\mathrm{Ar}^{+}+\mathrm{e}$ & $\begin{array}{l}2.1 \times 10^{-9} \\
\mathrm{~cm}^{3} \mathrm{~s}^{-1}\end{array}$ & [41] \\
\hline $\begin{array}{r}\text { (R7) } \operatorname{Ar}(4 p)+\operatorname{Ar}(4 p) \rightarrow \\
\mathrm{Ar}^{+}+\mathrm{Ar}+\mathrm{e}\end{array}$ & $\begin{array}{l}5.0 \times 10^{-10} \\
\mathrm{~cm}^{3} \mathrm{~s}^{-1}\end{array}$ & [41] \\
\hline $\begin{array}{l}\text { (R8) } \operatorname{dust}\left(\mathrm{Z}_{\mathrm{d}}\right)+\mathrm{Ar}^{*} \rightarrow \\
\quad \operatorname{dust}\left(\mathrm{Z}_{\mathrm{d}}+\gamma_{\mathrm{m}}\right)+\gamma_{\mathrm{m}} \mathrm{e}+\mathrm{Ar}_{0}\end{array}$ & $K_{m}^{d}$ & [22] \\
\hline (R9) $\quad$ e $\rightarrow$ wall & $R_{\mathrm{W}}$ & {$[34]$} \\
\hline $\begin{aligned} &(\mathrm{R} 10) \mathrm{e}+\operatorname{dust}\left(Z_{\mathrm{d}}\right) \rightarrow \rightarrow \\
& \operatorname{dust}\left(Z_{\mathrm{d}}-1\right)\end{aligned}$ & $K_{e}^{d}$ & [42] \\
\hline $\begin{array}{c}\text { (R11) } \mathrm{Ar}^{*}+\mathrm{C}_{2} \mathrm{H}_{2} \rightarrow \\
\mathrm{C}_{2} \mathrm{H}_{2}^{+}+\mathrm{Ar}+\mathrm{e}\end{array}$ & $\alpha k_{\mathrm{q}}$ & {$[27]$} \\
\hline
\end{tabular}

The reactions listed in table 2 , as well as the reactions (R2) $-(\mathrm{R} 8)$ in table 1 are used to calculate the 4 s resonant and $4 \mathrm{p}$ atoms densities.

The diffusion of metastable atoms to the walls is described by the rate $D_{m} / \Lambda^{2}$, where $D_{m} \approx 2.42 \times 10^{18} / n_{\mathrm{a}}$ is the metastable diffusion coefficient $[22,40], n_{\mathrm{a}}$ in $\mathrm{cm}^{-3}$ and $\Lambda=$ $1 / \sqrt{(\pi / L)^{2}+(2.405 / R)^{2}}$ is the effective diffusion length.

In the afterglow, the effective electron temperature is lower than in the on-period and the energetic tail of the EEPF is depleted because of deposition of high energetic electrons on the chamber walls and dust particles [43]. Thus, we assume that in the afterglow, the rate $K^{R I 6}$ is smaller (here, in 5 times) than in the on-period and the rate $K^{R 15}$ is $0.2 \int_{0}^{\infty} \sigma_{15}(\varepsilon) \sqrt{2 e \varepsilon / m_{e}} F(\varepsilon) \sqrt{\varepsilon} d \varepsilon$. For the on-period, the reaction rates are $K^{R 16}=2 \times 10^{-7} \mathrm{~cm}^{3} / \mathrm{s}$ [40] and $K^{R 15}=$ $\int_{0}^{\infty} \sigma_{15}(\varepsilon) \sqrt{2 e \varepsilon / m_{e}} F(\varepsilon) \sqrt{\varepsilon} d \varepsilon$, where $\sigma_{15}$ is the cross section for reaction $\mathrm{R} 15$ according to [44].

In our model, the time evolution of the effective electron temperature is found from the power balance equation:

$$
\frac{\partial}{\partial t}\left(\frac{3}{2} e n_{e} T_{\text {eff }}\right)=\frac{\left(P_{\mathrm{abs}}-P_{\text {loss }}\right)}{V},
$$

where $P_{\text {abs }}$ is the power absorbed in the plasma volume $V=$ $\pi R^{2} L$ and $\quad P_{\text {loss }}=P_{\text {coll }}+P_{w}+P_{d}$. Here, $P_{\text {coll }}=$ $e n_{e} n_{a} V \varepsilon_{c} K^{R 1}$ is the power loss due to elastic and inelastic collisions of electrons with $\mathrm{Ar}$ atoms, $\varepsilon_{c}$ is the collisional energy loss per electron-ion pair created [31] which is 
calculated using the cross-sections in [36]. $P_{w}=$ $e n_{i} u_{B}\left(2 \pi R^{2} \square_{L} \varepsilon_{w L}+2 \pi R L \square_{R} \varepsilon_{w R}\right)$ is the power loss due to charged particle fluxes to the walls, $\varepsilon_{w L}=\varepsilon_{i}+\varepsilon_{e}+V_{e l}$ is the energy loss per charged particle for the on-period in the direction parallel to the discharge axis, $\varepsilon_{w L}=\varepsilon_{i}+\varepsilon_{e}$ is the same for the off-period when the electron energy is much smaller than the electrode bias $V_{e l}$ and electrons do not deposit on the electrodes, $\varepsilon_{w R}=\varepsilon_{i}+\varepsilon_{e}$ is the energy loss per charged particle for the direction perpendicular to the discharge axis for the both on- and off-periods. $\varepsilon_{i}$ is the mean kinetic energy lost per ion [34] and $\varepsilon_{e}$ is the mean kinetic energy lost per electron [34]. $P_{\mathrm{d}}$ is the power loss to the dust particles [45]. The energy loss in collisions of electrons with $\mathrm{C}_{2} \mathrm{H}_{2}$ molecules is neglected in this model because of low $\mathrm{C}_{2} \mathrm{H}_{2}$ density.

Table 2. Production and loss processes of excited argon atoms used in the model, additionally to those in table 1.

\begin{tabular}{|c|c|c|}
\hline Reaction & Rate & Reference \\
\hline$(\mathrm{R} 12) \quad \mathrm{e}+\mathrm{Ar}_{0} \rightarrow \mathrm{Ar}_{\mathrm{m}}+\mathrm{e}$ & $K^{R 12}=f(F, \sigma)$ & [46] \\
\hline$(\mathrm{R} 13) \mathrm{e}+\mathrm{Ar}_{0} \rightarrow \mathrm{Ar}_{\mathrm{r}}+\mathrm{e}$ & $K^{R 13}=f(F, \sigma)$ & [46] \\
\hline$(\mathrm{R} 14) \quad \mathrm{e}+\mathrm{Ar}_{0} \rightarrow \operatorname{Ar}(4 \mathrm{p})+\mathrm{e}$ & $K^{R I 4}=f(F, \sigma)$ & [46] \\
\hline$(\mathrm{R} 15) \quad \mathrm{e}+\operatorname{Ar} \mathrm{m} \rightarrow \operatorname{Ar}(4 \mathrm{p})+\mathrm{e}$ & $K^{R 15}=f(F, \sigma)$ & [44] \\
\hline$(\mathrm{R} 16) \mathrm{e}+\mathrm{Ar}_{\mathrm{m}} \rightarrow \mathrm{Ar}_{\mathrm{r}}+\mathrm{e}$ & $K^{R 16}$ & [40] \\
\hline$(\mathrm{R} 17) \quad \mathrm{e}+\mathrm{Ar}_{\mathrm{r}} \rightarrow \mathrm{Ar}_{\mathrm{m}}+\mathrm{e}$ & $\begin{array}{c}9.1 \times 10^{-7} \\
\mathrm{~cm}^{3} \mathrm{~s}^{-1}\end{array}$ & [41] \\
\hline$(\mathrm{R} 18) \quad \mathrm{e}+\operatorname{Ar}_{\mathrm{r}} \rightarrow \operatorname{Ar}(4 \mathrm{p})+\mathrm{e}$ & $K^{R 18}=f(F, \sigma)$ & [44] \\
\hline$(\mathrm{R} 19) \mathrm{e}+\mathrm{Ar}_{\mathrm{m}} \rightarrow \mathrm{Ar}_{0}+\mathrm{e}$ & $K^{R 19}=f(F, \sigma)$ & {$[46,47]^{\mathrm{a}}$} \\
\hline$(\mathrm{R} 20) \mathrm{e}+\mathrm{Ar}_{\mathrm{r}} \rightarrow \mathrm{Ar}_{0}+\mathrm{e}$ & $K^{R 20}=f(F, \sigma)$ & {$[46,47]^{\mathrm{a}}$} \\
\hline$(\mathrm{R} 21) \mathrm{e}+\operatorname{Ar}(4 \mathrm{p}) \rightarrow \mathrm{Ar}_{0}+\mathrm{e}$ & $K^{R 21}=f(F, \sigma)$ & {$[46,47]^{\mathrm{a}}$} \\
\hline$(\mathrm{R} 22) \quad \mathrm{Ar}_{0}+\mathrm{Ar}_{\mathrm{m}} \rightarrow \mathrm{Ar}_{0}+\mathrm{Ar}_{0}$ & $\begin{array}{c}2.1 \times 10^{-15} \\
\mathrm{~cm}^{3} \mathrm{~s}^{-1}\end{array}$ & [41] \\
\hline$(\mathrm{R} 23) \quad \mathrm{Ar}_{\mathrm{r}} \rightarrow \mathrm{Ar}+\hbar \omega$ & $10^{5} \mathrm{~s}^{-1}$ & {$[41]$} \\
\hline$(\mathrm{R} 24) \quad \operatorname{Ar}(4 \mathrm{p}) \rightarrow \mathrm{Ar}+\hbar \omega$ & $3.2 \times 10^{7} \mathrm{~s}^{-1}$ & [41] \\
\hline$(\mathrm{R} 25) \quad \operatorname{Ar}(4 \mathrm{p}) \rightarrow \mathrm{Ar}_{\mathrm{m}}+\hbar \omega$ & $3.0 \times 10^{7} \mathrm{~s}^{-1}$ & [41] \\
\hline$(\mathrm{R} 26) \quad \operatorname{Ar}(4 \mathrm{p}) \rightarrow \mathrm{Ar}_{\mathrm{r}}+\hbar \omega$ & $3.0 \times 10^{7} \mathrm{~s}^{-1}$ & [41] \\
\hline (R27) $\mathrm{Ar}_{\mathrm{m}} \rightarrow \mathrm{Ar}_{0}$ (wall) & $D_{m} / \Lambda^{2}$ & {$[22,40]$} \\
\hline $\begin{aligned} \text { (R28) } \mathrm{Ar}^{*}+\mathrm{C}_{2} \mathrm{H}_{2} \rightarrow & \\
& \mathrm{C}_{2} \mathrm{H}+\mathrm{H}+\mathrm{Ar}+\mathrm{e} \\
\text { or } \quad & \mathrm{C}_{2} \mathrm{H}_{2}+\mathrm{Ar}+\hbar \omega\end{aligned}$ & $(1-\alpha) k_{\mathrm{q}}$ & [27] \\
\hline
\end{tabular}

${ }^{\text {a }}$ Obtained by applying the principle of detailed balancing to the cross section given in the cited reference.

We assume that the power is modulated by an ideal rectangular waveform

$$
P_{\mathrm{abs}}= \begin{cases}P_{\max } & \text { for } \quad(k-1) \tau \leq t<(k-1) \tau+\eta \tau, \\ 0 & \text { for } \quad(k-1) \tau+\eta \tau \leq t<k \tau,\end{cases}
$$

where $\tau$ is the full cycle period, $\eta$ is the duty cycle, and $k$ is a positive integer. $P_{\mathrm{abs}}=P_{\max }$ for the on-period and $P_{\mathrm{abs}}=0$ for the off-period. The time averaged absorbed power for the full cycle $<P_{\text {abs }}>$ is $\eta P_{\text {max }}$.

Considering the afterglow phase, we additionally assume that due to the production of energetic electrons in metastablemetastable collisions, super-elastic electron-metastable collisions and emission processes, the effective electron temperature cannot be smaller than the afterglow temperature $T_{a f t} \leq 0.15 \mathrm{eV}$, i.e. after its decay to reach $T_{a f t}, T_{\text {eff }}$ becomes time-independent. $T_{a f t}$ is a parameter chosen to match theoretical results and experimental data well.

The next equation of our system is the equation for the dust particle charge. The standard OML theory is usually used to describe the equilibrium dust charge in complex plasma. Nevertheless, several authors have shown that in the case of complex plasma OML theory fails to describe the dust charging in an appropriate way [48]. In order to describe the dust charging more accurately we (i) correct the ion current to the dust particles taking into account the ion neutral collisions [48] and (ii) include the additional processes which are relevant for dust charging [24, 25]. These processes, such as secondary electron emission by collisions of excited argon atoms with dust, have been neglected by OML theory. Nevertheless they play a significant role in dust charging/discharging processes under certain conditions [24, 25]. According to the assumptions (i) and (ii) the time dependent dust charge follows the equation

$$
\frac{\partial Z_{d}}{\partial t}=K_{i}^{d} n_{i}+\gamma_{m} K_{m}^{d}\left(n_{m}+n_{r}+n_{4 \mathrm{p}}\right)-K_{e}^{d} n_{e}
$$

Here $K_{i}^{d} \approx a_{d}^{2}\left(8 \pi e T_{i} / m_{i}\right)^{0.5}\left(1+\xi \tau+H \xi^{2} \tau^{2} \lambda_{S} n_{a} \sigma_{i a}\right)$ is the rate for collection of ions by dust particles, which accounts for ionneutral collisions in the sheath around a dust particle [48]. $\tau$ $=T_{\text {eff }} / T_{i}, \quad \xi=\left|Z_{d}\right| e /\left(a_{d} T_{\text {eff }}\right)$ and $\sigma_{i a} \approx 10^{-14} \mathrm{~cm}^{2}$ is the crosssection for ion-neutral collisions. The function $H$ has the following asymptotes: $H \sim 0.1$ for $0.1 \leq \beta \leq 10$; $H \sim \beta$ for $\beta<<1$ and $H \quad \sim \beta^{-2}(\ln \beta)^{3}$ for $\beta>>1$ [48], where $\beta=\left|Z_{d}\right| e /\left(\lambda_{s} T_{i}\right)$ and $\lambda_{s}$ is the screening length, which is of the same order as the Debye length [1].

The second term in the right-hand side of equation (5) accounts for electron emission in metastable-dust collisions. This process is not considered with acetylene impurity inside the plasma. The last term in the right-hand side of equation (5) describes the electron losses to the dust. When the plasma equilibrium is established, like at the end of the on- phase, the left -hand side of (5) becomes zero. Further, if the secondary electron emission is neglected when e.g. the density of argon reactive species is low, the equation (5) simplifies to the standard form used in OML theory.

The equations for each particle (electrons, excited argon atoms in metastable and $4 \mathrm{~s}$ and $4 \mathrm{p}$ resonance states), the power balance equation and the equation for dust charge are solved by using the DVODE package [49]. First, it is found a steadystate $\left(\frac{\partial}{\partial t}=0\right)$ solution of these equations corresponding to $P_{\text {abs }}$ $=P_{\max }$. Then, using this solution, the plasma parameters $\left(n_{\mathrm{e}}\right.$, $n_{\mathrm{m}}, n_{\mathrm{r}}, n_{4 \mathrm{p}},-Z_{\mathrm{d}}$ and $\left.T_{\text {eff }}\right)$ as a function of time are calculated for the case of pulsed plasma.

\section{Results and discussion}


Using the volume-averaged model, we have calculated plasma properties ( $n_{\mathrm{e}}, n_{\mathrm{m}}, T_{\mathrm{eff}}$ and $\left.Z_{\mathrm{d}}\right)$ as a function of time in dust-free and dusty plasma. The simulations have been carried out for the conditions typical to experiments on pure argon and argon/dust pulsed plasmas [22, 27], in particularly, for the same electron densities at the end of the on-period as in the experiments. The calculated time-dependencies for electron and metastable densities have been compared with the experimental values in $[22,27]$. We have also analyzed how these plasma parameters depend on the EEPF shape (which is characterized by the parameter $x$ ), the pulsing frequency and the duty cycle.

\subsection{Calculated time-dependencies of different plasma} properties and their comparison with experimental values

In the experiments $[22,27]$, a $13.56 \mathrm{MHz}$ capacitivelycoupled plasma discharge is ignited between two parallel plate $R=15 \mathrm{~cm}$ radius electrodes $L=7 \mathrm{~cm}$ separated. Both electrodes are powered by an RF signal with power in the range 10 to 80 $\mathrm{W}$ and delivered from an RF generator through a specially designed matching box. The working gas is either pure argon or argon-acetylene mixture at a continuous flow rate of $8 \mathrm{sccm}$ (argon) and $0.5 \mathrm{sccm}$ (acetylene), keeping the total pressure constant at $10 \mathrm{~Pa}$. The discharge is driven either in $\mathrm{CW}$ or in pulsed mode, with a variable pulsing frequency and a fixed duty cycle of 0.5 .

To generate the dust particles, it is necessary to use the acetylene precursor, which in some particular plasma conditions initiate the dust growth. In $\mathrm{CW}$ mode the nanoparticles are forming spontaneously after adding the acetylene to the argon. In the pulsed regime, however, it is necessary to run the discharge for at least $3 \mathrm{~min}$ and at pulsing frequencies above $700 \mathrm{~Hz}$ in order to form the dust [27]. Interrupting the $\mathrm{C}_{2} \mathrm{H}_{2}$ flow stops the nanoparticles' growth and for the next few minutes the particles stay imprisoned in the plasma bulk even at the lower pulsing frequency, thus forming the argon-dust plasma without $\mathrm{C}_{2} \mathrm{H}_{2}$. After forming the dust, the discharge is driven with the RF signal modulated by a rectangular signal frequency of $105 \mathrm{~Hz}$ with 0.5 duty cycle. Electron density is measured by microwave interferometry [16], while metastable density is deduced using laser absorption spectroscopy $[27,50]$. Both measurements give values integrated over the plasma length.

\subsubsection{Dust-free pulsed plasma}

Figure 1 illustrates the temporal behavior of the line-ofsight averaged electron density $(a)$ and $\mathrm{Ar}^{*} 1 \mathrm{~s}_{5}$ metastable density $(b)$ measured at the mid-plane between electrodes and the corresponding simulation results. Numerical results are obtained for $V_{\mathrm{el}}=58.0 \mathrm{~V}$ [51], $K_{\mathrm{p}}=1.24 \times 10^{-10} \mathrm{~cm}^{3} / \mathrm{s}, T_{\mathrm{aft}}=$ $0.15 \mathrm{eV}, T_{\mathrm{g}}=294 \mathrm{~K}$ and $x=2$. Note that figure 1 shows cycles taken in a middle of a long sequence, so before $0 \mathrm{~s}$ it is the end of another cycle. For both species, good quantitative agreement exists between the calculated and experimental time-dependencies. However, the agreement is better for the afterglow phase than for the on-period where the model gives a faster increase at the beginning of the on-period. In addition, there is a maximum in the calculated $n_{\mathrm{m}}$ at the beginning of the on-period that is not observed in the experiment.
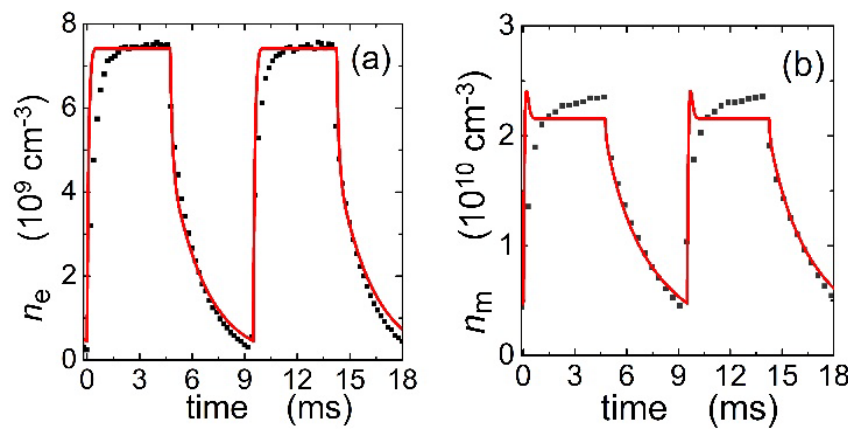

Figure 1. Electron $(a)$ and metastable $(b)$ densities as a function of time in a pure argon pulsed plasma. Solid lines model; black squares - experiment [22, 27, 33]. Zero time indicates the beginning of the on-period. The off-period (afterglow phase) starts at $4.75 \mathrm{~ms}$ and ends at $9.5 \mathrm{~ms}$.
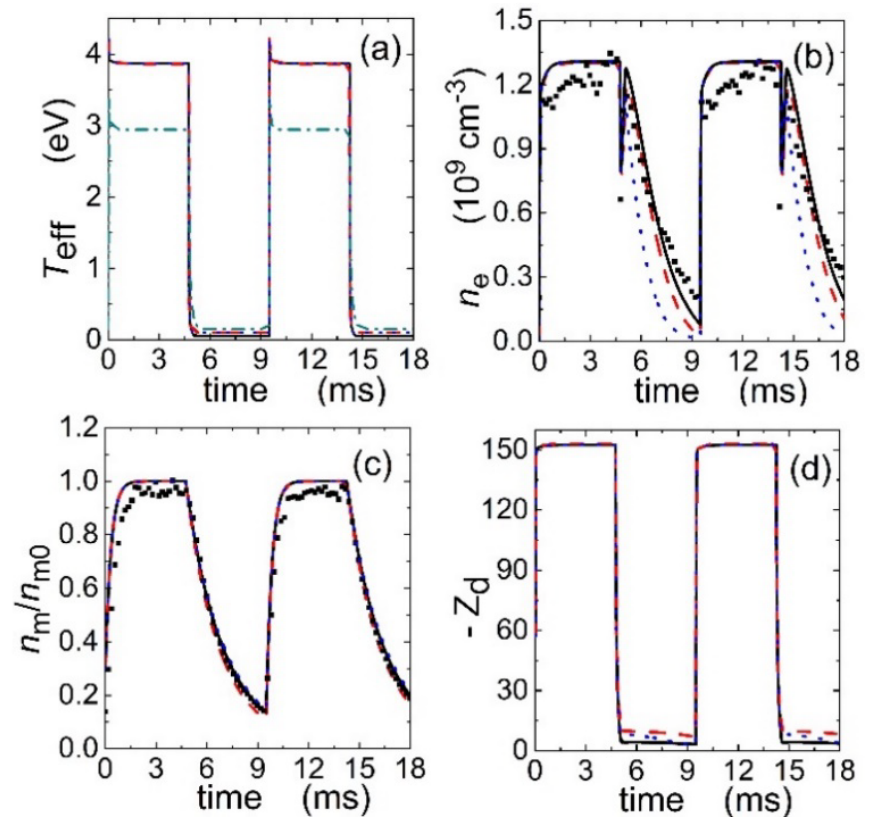

Figure 2. $T_{\text {eff }}(a), n_{\mathrm{e}}(b), n_{\mathrm{m}} / n_{\mathrm{m} 0}(c)$ and $-\mathrm{Z}_{\mathrm{d}}(d)$ in an argon/dust pulsed plasma. (i) The solid and dotted curves are calculated taking into account secondary electrons in metastable-dust collisions with $n_{\mathrm{m} 0}=9.82 \times 10^{10} \mathrm{~cm}^{-3}, T_{\text {aft }}=0.05 \mathrm{eV}$ or $T_{\text {aft }}=0.1$ $\mathrm{eV}$ for the solid and dotted curves, respectively; (ii) dashed curves - electrons are generated in collisions of metastable atoms with acetylene molecules with $n_{\mathrm{m} 0}=9.64 \times 10^{10} \mathrm{~cm}^{-3}$ and $T_{\text {aft }}=0.1 \mathrm{eV}$. For the comparison, the dash-dotted curve in figure $(a)$ represents $T_{\text {eff }}$ in a pure argon pulsed plasma. The black squares in figures $(b)$ and $(c)$ are experimental data from $[22,27] . n_{\mathrm{m} 0}=1.75 \times 10^{11} \mathrm{~cm}^{-3}$ is a measured value reported in $[22,27]$. 
During the on-period, $n_{\mathrm{m}}$ depends on $n_{\mathrm{e}}$ and $T_{\text {eff }}$ strongly, because the formation of metastable atoms occurs mainly in collisions of electrons with argon atoms in the ground state. During the initial stage of the on-period, a sharp spike in $T_{\text {eff }}$ is observed (figure 2(a)). This is due to a small number of electrons left in the discharge at the end of the previous afterglow phase. At the beginning of the new on-period, the $\mathrm{RF}$ power is absorbed by this small number of electrons inducing their strong heating and thus a higher $T_{\text {eff }}$ explaining also the sharp spike in $n_{\mathrm{m}}$. Note that spikes in the electron temperature and density of excited species at the very beginning of the on-period are observed in a few numerical and experimental studies on pulsed plasmas in various reactor geometries and plasma conditions [52 - 54].

In the afterglow phase, $n_{\mathrm{e}}$ and $n_{\mathrm{m}}$ monotonically decrease mainly due to diffusion to the chamber walls [22]. Nevertheless, the loss of metastable atoms in collisions with electrons prevails in the early afterglow [22].

\subsubsection{Dusty plasma}

The presence of dust in plasma introduces additional production and loss processes for electrons and $\mathrm{Ar}$ metastables. Two additional processes are analyzed separately in two different scenarios: (i) secondary electrons are generated by metastable-dust collisions; (ii) production of electrons by collisions of metastable atoms with acetylene impurity remaining in the plasma from the dust formation stage [27]. In the case (i), we use $\gamma_{\mathrm{m}}=0.2$ and two different afterglow temperatures $T_{\text {aft }}(0.05 \mathrm{eV}$ and $0.1 \mathrm{eV})$. In the case (ii), $T_{\text {aft }}$ is $0.1 \mathrm{eV}$ and the acetylene density $n_{\mathrm{C} 2 \mathrm{H} 2}$ is $10^{11} \mathrm{~cm}^{-3}$, according to the figure 11(a) in [27]. In both cases, we assume that $a_{\mathrm{d}}=50 \mathrm{~nm}, n_{\mathrm{d}}=3.0 \times 10^{7} \mathrm{~cm}^{-3}, T_{\mathrm{g}}=366 \mathrm{~K}[22], K_{\mathrm{p}}=1.24$ $\times 10^{-10} \mathrm{~cm}^{3} / \mathrm{s}, V_{\mathrm{el}}=34.0 \mathrm{~V}[51]$ and $x=2$.

The calculated $n_{\mathrm{e}}$ and $n_{\mathrm{m}}$ follow relatively well the experimental values in the case (ii) and the case (i) with $T_{\text {aft }}=$ $0.05 \mathrm{eV}$. However, in the late afterglow, the decay time for electron density in the case (ii) is slightly shorter than in the case (i) with $T_{\text {aft }}=0.05 \mathrm{eV}$ (figure 2(b)). This is due to larger $T_{\text {aft }}$ and, as a consequence, larger loss of electrons due to diffusion to the walls in the former case. At larger $T_{\text {aft, }}$, the electron flux to dust particles enhances, increasing the absolute value of dust charge number $\left|Z_{\mathrm{d}}\right|$. Therefore, $\left|Z_{\mathrm{d}}\right|$ in late the afterglow in the case (i) and $T_{\text {aft }}=0.05 \mathrm{eV}$ is smaller than in the case (ii) (figure 2(d)). In addition, the secondary electron emission in metastable -dust collisions is stronger in the case (i), which reduces the negative charge on dust particles in comparison with case (ii). If $T_{\text {aft }}$ is the same in both cases (i) and (ii), the loss of electrons on dust particles in the afterglow is larger in the case (i) because of smaller $\left|Z_{d}\right|$ (figure $2(d)$ ) due to secondary emission. Because of larger electron loss on dust particles, in the off-period $n_{\mathrm{e}}$ drops faster in the case (i) than in the case (ii) for the same $T_{\text {aft }}$ (figure $2(b)$ ). In both cases (i) and (ii), the calculated metastable densities are nearly 1.8 times smaller than the densities measured in the experiment. This difference may be explained by simplifications introduced in the model, which will be discussed later.
Insertion of dust particles into a plasma during the onperiod is accompanied by a decrease in the electron density (figures $2(b)$ and $1(a)$ ) and an increase in the effective electron temperature (figure 2(a)) and the density of metastable argon atoms (figures $2(c)$ and $1(b)$ ). Due to the loss of electrons and metastable atoms on dust particles, the calculated electron and metastable densities increase more slowly at the very beginning of the on-period in the presence of dust particles compared to the dust-free case (figures 1 and 2).

In the on-period, the density of ions $\left(n_{i}=n_{e}+n_{d}\left|Z_{d}\right|\right)$ in the dusty plasma is smaller than $n_{i}\left(=n_{e}\right)$ in the dust-free case (figures $1(a)$ and 3 ). This decrease in the ion density in the dusty plasma, comparing with the dust-free plasma, is due to the decrease of electron density. In the beginning of the offperiod, the density of ions in the dusty plasma drops faster than $n_{e}$ in the dust-free and dusty plasma. The significant number of $\mathrm{Ar}^{+}$ions is captured by negatively charged dust particles, which causes this fast ion decay. The term $K_{i}^{d} n_{i}$ in equation (5) can be essentially larger than the term $K_{e}^{d} n_{e}$ (see figure $4(b)$ of [22]) because the rate $K_{e}^{d}$ depends strongly on $T_{\text {eff }}$ (for example, if the Maxwellian is assumed this dependence is nearly exponential), while the rate $K_{i}^{d}$ depends weakly of the effective electron temperature. The rate $K_{i}^{d}$ decreases with decreasing $\left|Z_{d}\right|$ and, therefore, for most of the off-period, when $\left|Z_{d}\right|$ is small (figure $2(d)$ ), the decay time for $n_{i}$ is nearly the same as that for $n_{e}$ in the late afterglow (figures 2(b) and $3)$.

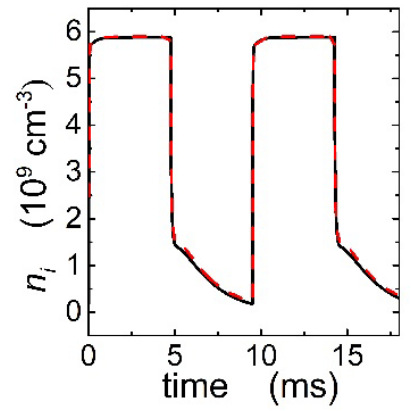

Figure 3. $n_{\mathrm{i}}$ as a function of time. The solid and dashed curves are obtained for the same conditions as the solid and dashed curves in figure $2(b)$, respectively.

In the on-period, the production of electrons occurs mainly due to electron-atom collisions, while this production process can be neglected in the afterglow. In the off-period, the main processes of electron generation are collisions of metastable atoms with other species (acetylene molecules or dust particles). In the beginning of the afterglow phase, when the metastable density is high enough, the electron production in metastable collisions is dominating the loss of electrons on the walls and dust particles, which causes a peak of $n_{\mathrm{e}}$ in the afterglow (at $t \sim 5.2 \mathrm{~ms}$ in figure $2(b)$ ) $[22,27]$.

\subsection{Effects of variation in the EEPF shape}

Next, we analyze how a variation in the EEPF shape affects the plasma properties. For that, we vary the parameter 
$x$ in equation (1), while other process parameters, including $n_{\mathrm{e}}$ at the end of the on-period (at $t=4.75 \mathrm{~ms}$ in figure $4(a)$ ), remain fixed.

\subsubsection{Dust-free pulsed plasma}
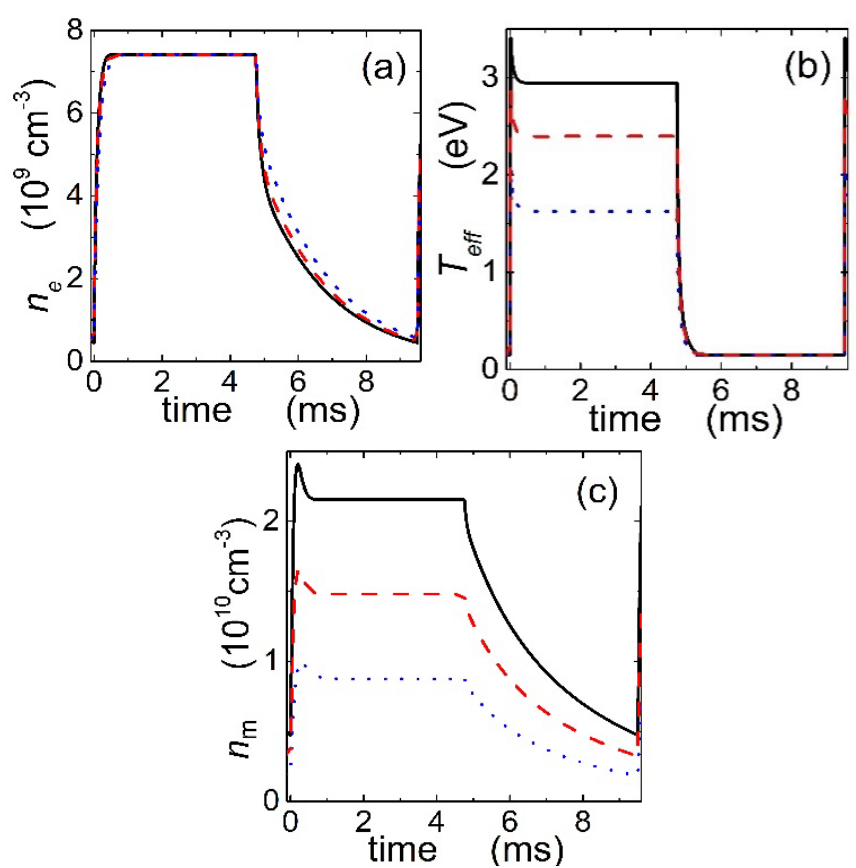

Figure 4. Calculated $n_{\mathrm{e}}(a), T_{\text {eff }}(b)$ and $n_{\mathrm{m}}(c)$ as functions of time in a pure argon pulsed plasma for different shapes of the EEPF: Solid line $-x=2$, dashed line $-x=1.5$ and dotted line $-x=1$.

In figure 4, the time-dependencies for $n_{\mathrm{e}}, T_{\text {eff }}$ and $n_{\mathrm{m}}$ are shown for different $x$ in the case of pure argon plasma. The external conditions and assumptions are the same as in figure 1. One can see in figure $4(b)$ that $T_{\text {eff }}$ in the on-period becomes higher with increasing $x$ because the electron energy probability function becomes more convex [55]. Due to the temperature increase, the densities of excited argon atoms, including metastables, increase too (figure 4(c)). Meanwhile, the density of electrons in the afterglow becomes smaller with increasing $x$ (figure $4(a)$ ) due to an enhancement of the electron loss on the walls because of the increase of the Bohm velocity. In particular, $u_{B}=\sqrt{e T_{\text {eff }} / m_{i}}$ and $u_{B}=$ $\sqrt{3 \times 0.457 e T_{\mathrm{eff}} / m_{i}}$ [55] for $x=1$ and $x=2$, respectively. Here, at the beginning of the afterglow, $T_{\text {eff }}(x=2)>T_{\text {eff }}(x=1)$, while these temperatures are equal to each other for large afterglow times.

For the case $x=1$, the calculated metastable density during most of the on-period is essentially smaller than that obtained in the experiment (figures $1(b)$ and $4(c)$ ). According to the model the Druyvesteyn EEPF leads to the better agreement between calculated and measured values of $n_{\mathrm{m}}(t)$ than Maxwellian EEPF.

\subsubsection{Dusty pulsed plasma}

In figure $5, T_{\text {eff, }} n_{\mathrm{m}}$ and $n_{\mathrm{e}}$ as functions of time are shown for the case of dusty plasma and different $x$. The input parameters are $n_{\mathrm{e}}(t=4.75 \mathrm{~ms})=1.3 \times 10^{9} \mathrm{~cm}^{-3}, n_{\mathrm{C} 2 \mathrm{H} 2}=10^{11} \mathrm{~cm}^{-3}$ and $T_{\text {aft }}=0.1 \mathrm{eV}$, and the production of electrons by electronatom, metastable-metastable and metastable-acetylene collisions is assumed. Other parameters and assumptions, are the same as in figure 2 .
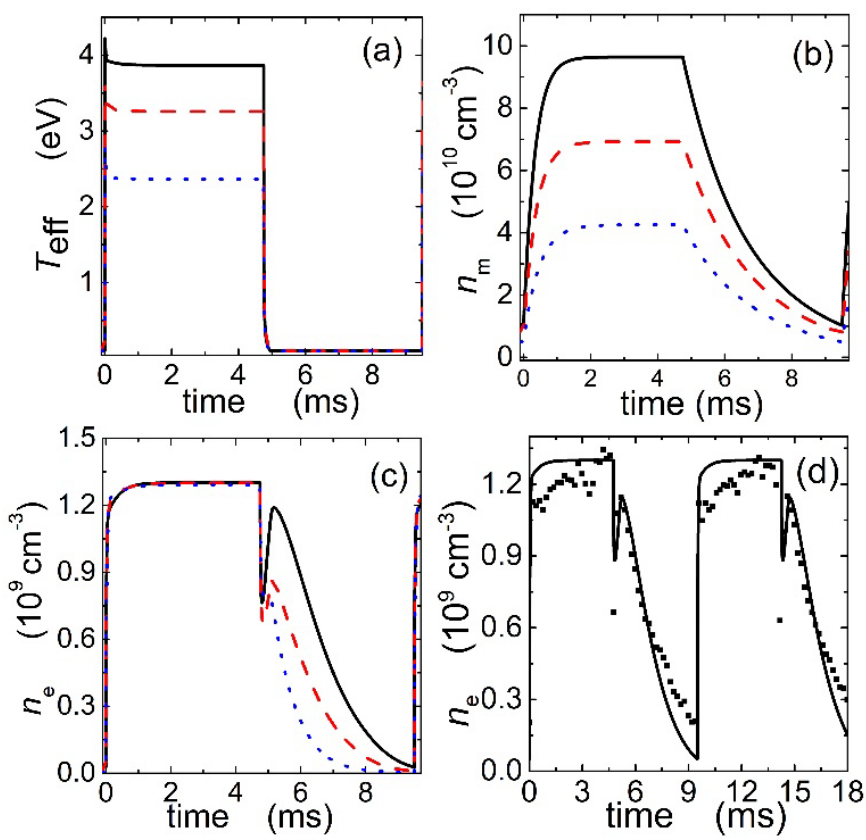

Figure 5. $T_{\mathrm{eff}}(a), n_{\mathrm{m}}(b)$ and $n_{\mathrm{e}}(\mathrm{c})$ for different $x: 2$ (solid line), 1.5 (dashed line) and 1.0 (dotted line). (d) $n_{\mathrm{e}}$ calculated (solid line) for $x=1, K_{\mathrm{p}}=6.2 \times 10^{-10} \mathrm{~cm}^{3} / \mathrm{s}, T_{\mathrm{aft}}=0.05 \mathrm{eV}, n_{\mathrm{C} 2 \mathrm{H} 2}$ $=10^{11} \mathrm{~cm}^{-3}$ and the same other conditions as in figure 2 . The black squares in figure $(d)$ are experimental data from [22, 27].

As in the case of the Druyvesteyn EEPF (figures 1 and 2), the density of metastable atoms and the effective electron temperature during the on-period are higher in a pulsed dusty plasma than in a dust-free plasma (figures 4 and 5) for the same $x(=1$ or 1.5$)$. As in the case of dust-free pulsed plasma, $n_{\mathrm{m}}$ and $T_{\text {eff }}$ increase when $x$ becomes larger (figures $5(a)$ and $5(b))$. The increase of $n_{\mathrm{m}}$ with $x$ is accompanied by an enhancement of electron production in metastable-metastable collisions and collisions of metastable atoms with $\mathrm{C}_{2} \mathrm{H}_{2}$ molecules and, as a result, the electron density in the offperiod increases also (figure 5(c)).

Note that the time-dependence for $n_{\mathrm{e}}$ in the case $x=1$ (figure 5(c)), differs essentially from the experimental data (figure 2(b)). Moreover, at the end of the on-period, the calculated metastable densities for $x=1$ and $x=1.5$ (figure $5(b)$ ) are much smaller than the measured one $\left(1.75 \times 10^{11} \mathrm{~cm}^{-3}\right)$.

The time-dependence for $n_{\mathrm{e}}$ calculated in the case $x=1$ may agree well with the experimental data if one assumes that the metastable pooling process is stronger. Figure $5(d)$ compares the experimental data and the model results for the 
$x=1$ and $K_{\mathrm{p}}=6.2 \times 10^{-10} \mathrm{~cm}^{3} / \mathrm{s}$ instead of $1.24 \times 10^{-10} \mathrm{~cm}^{3} / \mathrm{s} .\left(K_{\mathrm{p}}\right.$ $=6.2 \times 10^{-10} \mathrm{~cm}^{3} / \mathrm{s}$ is commonly used in the literature $[22,32]$.) Although the overall agreement is better, the stronger metastable pooling leads to much shorter electron decay time in the afterglow than expected, since $n_{\mathrm{e}} \sim n_{\mathrm{m}}{ }^{2}$. Recent analysis [27] suggests the metastable pooling rate by order of magnitude smaller than the rate commonly used in the literature $[22,32]$. In this way, by using new smaller pooling rate and Druyvesteyn EEPF the properties of argon/dust pulsed plasma are described in better agreement with the experiment.

\subsection{Effects of variation in the pulsing frequency}

Properties of pulsed plasmas also depend on the pulsing frequency $v_{\mathrm{p}}=1 / \tau[52,53]$. Therefore, we analyze how a variation in the pulsing frequency affects plasma properties.

\subsubsection{Dust-free plasma}

First consider the case of dust-free plasma. In figure 6 , the electron and metastable densities and effective electron temperature as functions of time in a dust-free pulsed plasma are shown for different pulsing frequencies: $210,840,1680$ and $6720 \mathrm{~Hz}$.
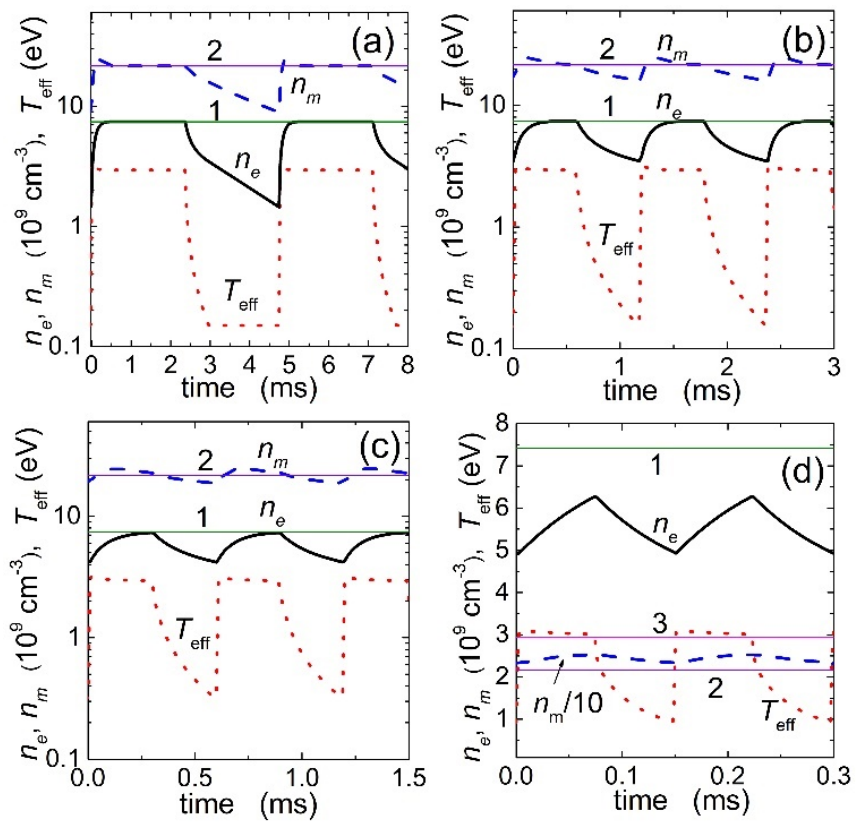

Figure 6. $n_{\mathrm{e}}$ (solid line), $n_{\mathrm{m}}$ (dashed line) and $T_{\text {eff }}$ (dotted line) for the dust-free plasma and the different pulsing frequencies: $210(a), 840(b), 1680(c)$ and $6720(d)$ Hz. The other conditions are the same as in figure 1 . The lines 1 and 2 correspond, respectively, to $n_{\mathrm{e}}$ and $n_{\mathrm{m}}\left(n_{\mathrm{m}} / 10\right.$ for figure $\left.6(d)\right)$ calculated for the CW discharge with $P_{\mathrm{abs}}=P_{\max }$, where $P_{\max }$ $=8.7 \mathrm{~W}$. The line 3 in figure $6(d)$ corresponds to $T_{\text {eff }}$ in the CW case.
It can be seen from figure 6 that $n_{\mathrm{e}}$ during the on-period can become equal to the density in the $\mathrm{CW}$ discharge, in which $P_{\mathrm{abs}}=P_{\max }$, if the pulsing frequency is not high (here, $v_{\mathrm{p}} \leq$ $1680 \mathrm{~Hz}$ ). For large $v_{\mathrm{p}}$ values (for example, $6720 \mathrm{~Hz}$ ), the maximum electron density during the on-period is less than $n_{\mathrm{e}}$ in the CW discharge due to short duration of the on-period (figure $6(d)$ ). The maximum effective temperature in the onperiod of the pulsed plasmas is also higher than the temperature corresponding to the $\mathrm{CW}$ mode ( $T_{\text {eff }}$ is nearly 2.95 $\mathrm{eV})$. With an increase in the pulsing frequency, the minimum values of $n_{\mathrm{e}}, n_{\mathrm{m}}$ and $T_{\text {eff }}$ (if the minimum temperature is larger than $0.15 \mathrm{eV}$ ) become larger because of decreasing the offtime (figure 6). Since at large values of $v_{\mathrm{p}}$ the effective temperature during most on-time is higher than that in the $\mathrm{CW}$ discharge, the metastable density during the full cycle in the pulsed plasma is larger than $n_{\mathrm{m}}$ in the CW case (figure $6(d)$ ).

\subsubsection{Dusty plasma}
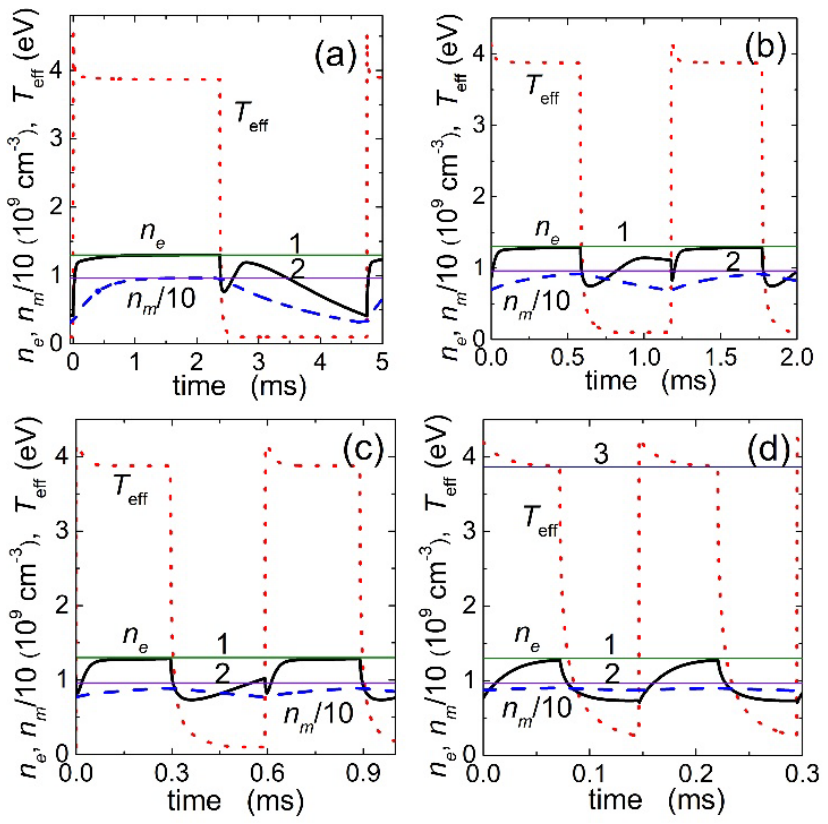

Figure 7. $n_{\mathrm{e}}$ (solid line), $n_{\mathrm{m}} / 10$ (dashed line) and $T_{\text {eff }}$ (dotted line) as a function of time in Ar/dust pulsed plasma at different $v_{\mathrm{p}}$ : $210(a), 840(b), 1680(c)$ and $6720(d) \mathrm{Hz}$. The line 1 describes $n_{\mathrm{e}}$, the line 2 describes $n_{\mathrm{m}} / 10$ and the line 3 (only in (d) $T_{\text {eff }}$ for the CW plasma with $P_{\mathrm{abs}}=P_{\max }$. Here, $P_{\max }=$ $12.6 \mathrm{~W}$ and the other conditions are the same as in figure 2.

Next consider how a variation of the pulsing frequency affects the properties of an argon pulsed plasma if the plasma contains dust particles. In figure 7 , the calculated timedependencies for $n_{\mathrm{e}}, n_{\mathrm{m}}$ and $T_{\text {eff }}$ are shown for different $v_{\mathrm{p}}$. Again, it is assumed that electrons are produced in electronatom, metastable-metastable and metastable-acetylene collisions. According to figure 7 , if the pulsing frequency is low (for all the cases in figure 7, except for the $v_{\mathrm{p}} 6.72 \mathrm{kHz}$ ) and $P_{\mathrm{abs}}=P_{\max }$ the electron densities in pulsed and $\mathrm{CW}$ plasma are equal for the most of the on-period, like in the dustfree plasma. 
Note that for high pulsing frequencies, the metastable density in the pulsed dusty plasma during the full cycle is smaller than in the CW dusty plasma (figures $7(c)$ and $7(d)$ ). This is opposite to the dust-free plasma, where $n_{\mathrm{m}}$ in a pulsed plasma with large $v_{\mathrm{p}}$ is higher than the $\mathrm{CW}$ metastable density (figure $6(d)$ ). Since in both the dust and dust-free pulsed plasmas with high $v_{\mathrm{p}}$ the electron density for the most of the on-period is smaller than the electron density in the $\mathrm{CW}$ plasma, the difference in $n_{\mathrm{m}}$, in our opinion, is due to a difference in $T_{\text {eff. Indeed, }} T_{\text {eff }}$ in the pulsed dusty plasma varies faster than in the dust-free plasma because of the electron energy loss on dust particles [22].

At high pulsing frequencies (for example, at $6720 \mathrm{~Hz}$ ), $T_{\text {eff }}$ in the second half of the on-period is nearly the same as that in the CW dusty plasma (figure $7(d)$ ). In the case of pure argon plasma, the effective temperature varies more slowly during the on-period, and at large $v_{\mathrm{p}}, T_{\text {eff }}$ during most of the on-period is higher than the temperature of the CW discharge (figure $6(d)$ ). Moreover, for the same high pulsing frequencies, $T_{\text {eff }}$ in the off-period decreases more rapidly and to lower values, if dust particles are in the plasma. Therefore, the production of metastable atoms in electron-atom collisions in the afterglow lasts longer in the dust-free plasma.

There is another difference between pulsed dust-free and dusty plasmas. At some $v_{\mathrm{p}}$, the electron density decreases rapidly in the very beginning of the on-period in the dusty case (figures $7(b)$ and $7(c)$ ). In our opinion, the increase of $T_{\text {eff }}$ at the beginning of the on-period leads to the higher electron flux to the dust particles and the rapid decrease of $n_{\mathrm{e}}$ (figure $8(a)$ ).
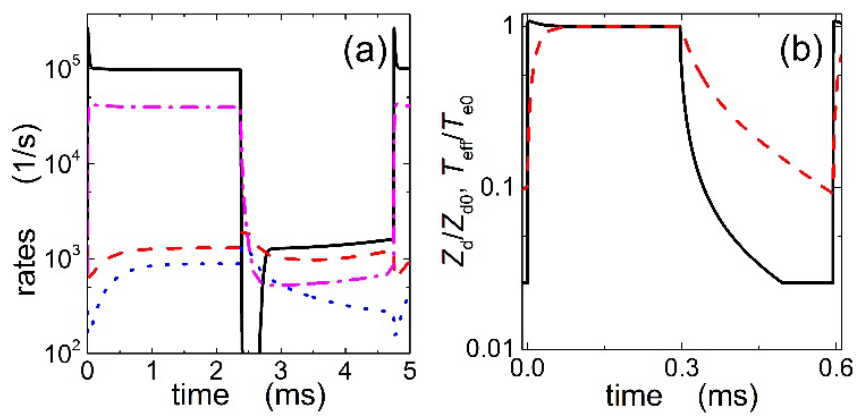

Figure 8. (a) The rates $K_{e}^{d} n_{d}$ (solid line), $n_{i} k_{w i} / n_{e}$ (dashdotted line), $\alpha k_{\mathrm{q}}\left(n_{\mathrm{m}}+n_{\mathrm{r}}+n_{4 \mathrm{p}}\right) n_{\mathrm{C} 2 \mathrm{H} 2} / n_{\mathrm{e}}$ (dashed line) and $K_{p} n_{m}^{2} /$ $n_{e}$ (dotted line) as functions of time for the $210 \mathrm{~Hz}$ pulsed dusty plasma. (b) The normalized dust charge (dashed line) and the normalized effective electron temperature (solid line) for the $1680 \mathrm{~Hz}$ pulsed dusty plasma. Here, $\mathrm{Z}_{\mathrm{d} 0}=-152.64$ and $T_{\mathrm{e} 0}=3.88 \mathrm{eV}$, and the other external conditions are the same as in figure 7 .

Our calculations show that the rapid decrease of $n_{\mathrm{e}}$ in the beginning of the on-period is not observed at low $v_{\mathrm{p}}$ (figure $7(a)$ ), and the decrease is small for high $v_{\mathrm{p}}$ (figure $7(d)$ ). We do not observe the decrease of $n_{\mathrm{e}}$ at low $v_{\mathrm{p}}$ because of small electron density at the end of the off-period. At smaller $n_{\mathrm{e}}$ in the end of the afterglow phase, the overshoot in $T_{\text {eff }}$ in the beginning of the on-period is sharper [52]. As a consequence, at low $v_{\mathrm{p}}$, production of electrons due to ionization grows very rapidly when the power is turned on, and the production dominates the total electron loss at the beginning of the onperiod. For the $6720 \mathrm{~Hz}$, the decrease of electron density is smaller compared with the $840 \mathrm{~Hz}$ and $1680 \mathrm{~Hz}$ (figure 7) because of smaller rate $K_{e}^{d} n_{d}$ at the end of the off-period, and, consequently, smaller electron losses in the very beginning of the on-period. $\left(K_{e}^{d} n_{d}=0.7,145.8\right.$ and $1282.8 \mathrm{~s}^{-1}$ at the end of the off-period for the frequencies 6720,1680 and $840 \mathrm{~Hz}$, respectively). For large $v_{\mathrm{p}}(\geq 1680 \mathrm{~Hz})$, the loss of electrons on the walls described by the rate $n_{i} k_{w i} / n_{e}$ and the electron production in collisions of excited atoms with acetylene molecules (described be the rate $\left.\alpha k_{\mathrm{q}}\left(n_{\mathrm{m}}+n_{\mathrm{r}}+n_{4 \mathrm{p}}\right) n_{\mathrm{C} 2 \mathrm{H} 2} / n_{\mathrm{e}}\right)$ and in metastable-metastable collisions (described by the rate $K_{p} n_{m}^{2} / n_{e}$ ) are more intensive than the loss of electrons on the dust particles during the most of the off-period (figure $8(a)$ ).

The rate $K_{e}^{d} n_{d}$ decreases rapidly in the beginning of the off-period due to the decrease of the effective electron temperature, which drops faster than $\left|Z_{\mathrm{d}}\right|$ (figure $8(b)$ ). The decrease of $K_{e}^{d} n_{d}$ in the beginning of the off-period takes place until $T_{\text {eff }}$ reaches the afterglow temperature $T_{\text {aft }}$. After that, the loss rate characterizing electron deposition on dust particles increases with time, since the amount of negative charge on dust particles decreases (figure 8).

\subsection{Effects of variation in duty ratio on the plasma} properties
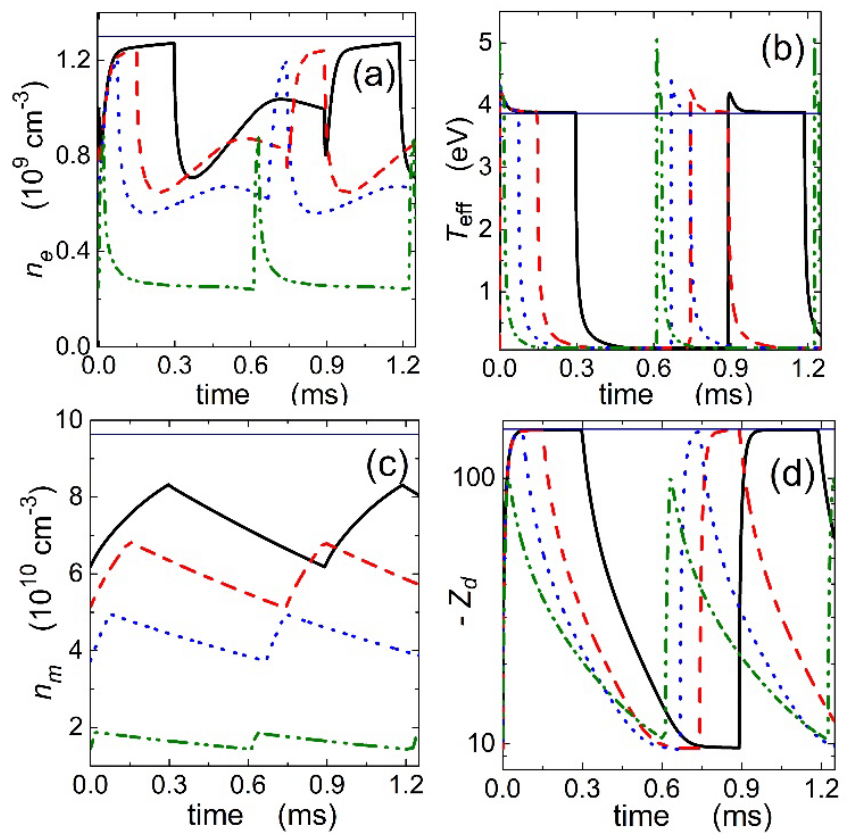

Figure 9. $n_{\mathrm{e}}(a), T_{\mathrm{eff}}(\mathrm{b}), n_{\mathrm{m}}(c)$ and $-Z_{\mathrm{d}}(d)$ as a function of time in argon/dust pulsed plasma for different duty cycles: $1 / 3$ (solid line), 1/5 (dashed line), 1/9 (dotted line) and 1/33 (dashdot-dotted line). The duration of the off-period is $19 / 32 \mathrm{~ms}$. The horizontal lines are for the $\mathrm{CW}$ mode with $P_{\mathrm{abs}}=P_{\mathrm{max}}$. The other external conditions are the same as for figure 7 .

In figure 9, the time-dependencies for $n_{\mathrm{e}}$ (figure $9(a)$ ), $T_{\text {eff }}$ (figure $9(b)), n_{\mathrm{m}}$ (figure $9(c)$ ) and $-Z_{\mathrm{d}}($ figure $9(d)$ ) are shown 
for the argon dusty plasma and different duty cycles: $1 / 3,1 / 5$, $1 / 9$ and $1 / 33$. For the sake of comparison, the same timedependencies are also presented in figure 9 for the $\mathrm{CW}$ mode.

One can see from figure $9(a)$ that the maximum electron density decreases with decreasing $\eta$ (figure $9(a)$ ). The decrease of the maximum electron density is accompanied by a decrease of $n_{\mathrm{e}}$ at the end of the afterglow stage. Since with decreasing $\eta$, the input RF power in the beginning of the onperiod is absorbed by a more limited number of electrons (smaller $n_{\mathrm{e}}$ ), the overshoot in $T_{\text {eff }}$ becomes sharper (figure $9(b))$. Due to the decrease of $n_{\mathrm{e}}$, the metastable density becomes smaller when $\eta$ decreases (figure 9(c)).

With decreasing the duty cycle, the rapid decrease of $n_{\mathrm{e}}$ at the beginning of the on-period becomes less pronounced and is not observed at small $\eta$ (for example, for the $\eta=1 / 33$ in figure $9(a)$ ). In our opinion, this is due to more rapid increase of $T_{\text {eff }}$ at the beginning of the on-period. Indeed, since the effective electron temperature increases more rapidly with a decrease of $\eta$, the ionization rate for the production of electrons in electron-atom collisions also grows faster and, as a result, for small $\eta$ the electron production dominates the total electron loss at the beginning of the on-period.

For small $\eta$, the amount of negative charges on dust particles at the end of the on-period decreases when the onperiod becomes shorter (figure $9(d)$ ). This is due to the decrease of the maximum electron density (figure $9(a)$ ). The decrease of $\eta$ is followed by the decrease of ion density too (the electron and ion densities are connected through the quasi -neutrality condition (3)). Moreover, for the small duty cycles the decay time of $\left|Z_{\mathrm{d}}\right|$ in the afterglow phase is longer than for large $\eta$ (figure $9(d)$ ). The decrease of ion density leads to the lower ion flux to the dust and the slower discharging of the dust particles for the smaller $\eta$.

We also studied how properties of a dust-free pulsed plasma depend on the duty cycle (the results are not shown here). It was found that, similarly to the dusty plasma, for small $\eta$ the electron and metastable densities in the beginning of the off-period and $n_{\mathrm{e}}$ at the end of the afterglow become smaller with decreasing the duty cycle. With a decrease of $\eta$, the overshoot in $T_{\text {eff }}$ also becomes sharper.

\section{Summary}

In summary, we have developed a spatially-averaged model for an argon/dust pulsed plasma. Using the model, it has been shown how the plasma properties depend on the shape of electron energy probability function, the pulsing frequency and the duty cycle. The properties of a pure argon pulsed plasma have been also analyzed. The studies have been carried out for the external conditions close to the experimental conditions in $[22,27]$. The calculated timedependencies for electron and metastable atom densities have been compared with those obtained experimentally for dustfree and dusty plasma and found to be in a good qualitative agreement (figures 1 and 2).

In particular, it has been shown that for the same $n_{\mathrm{e}}$ at the end of the on-period, the effective electron temperature is higher and, as a result, the metastable atom density is larger in the case of Druyvesteyn EEPF $(x=2)$ than in the Maxwellian case $(x=1)$. This is the case for both dust-free and dusty plasmas (figures 4 and 5). However, with increasing $x$, the electron density in the late afterglow decreases in the dust-free plasma (figure 4(a)), while it becomes larger in the dusty plasma (figure 5(c)). This decrease in the dust-free plasma is mainly due to an increase of the electron flux to the walls (because of increasing the Bohm velocity). The increase of $n_{\mathrm{e}}$ with $x$ in the afterglow dusty plasma is due to an enhancement of production of electrons in collisions of metastable atoms with other species (as $n_{\mathrm{m}}$ grows with $x$ ) such as acetylene molecules remaining in the plasma from the dust formation stage. For both $n_{\mathrm{d}}=0$ and $n_{\mathrm{d}} \neq 0$, the calculated metastable densities agree better in magnitude with the measured $n_{\mathrm{m}}$ for the Druyvesteyn than in the Maxwellian EEPF. The new proposed argon metastable pooling rate and the new Penning branching ratio for $\mathrm{Ar}^{*}-\mathrm{C}_{2} \mathrm{H}_{2}$ quenching [27] supports this finding.

The pulsed plasma properties depend on the pulsing frequency. The metastable density in a dust-free pulsed plasma with high $v_{\mathrm{p}}$ is larger than in the $\mathrm{CW}$ plasma for the same $P_{\text {abs }}$ and $P_{\max }$ powers (figure $6(d)$ ). This is opposite to the dusty plasma, where $n_{\mathrm{m}}$ at high $v_{\mathrm{p}}$ is smaller than the metastable density in the $\mathrm{CW}$ mode (figure $7(d)$ ). As the effective electron temperature variates faster the collection of electrons by dust particles becomes larger and the $n_{\mathrm{m}}$ smaller [22]. Our calculations have also shown that the electron density in a pulsed dusty plasma may decrease rapidly at the beginning of the on-period because of the rapid enhancement of collection of electrons by dust particles (figures $7(b)$ and $7(c))$

The properties of pulsed dusty plasma also depend essentially on the duty cycle $\eta$. The electron densities at the very beginning and at the end of the off-period decrease with a decrease of $\eta$ (figure $9(a)$ ). With decreasing $n_{\mathrm{e}}$ at the end of the off-period, the effective electron temperature increases more rapidly in the beginning of the next on-period (figure $9(b))$. The rapid increase of $T_{\text {eff }}$ is accompanied by an enhanced production of electrons (in electron-atom collisions). This larger electron production dominates the total electron loss at small $\eta$ resulting in the disappearance of the rapid decrease of $n_{\mathrm{e}}$ at the beginning of the on-period (for example, for $\eta=1 / 33$ in figure $9(a)$ ). The rapid decrease in $n_{\mathrm{e}}$ is also absent when the off-period is long (figures $2(b)$ and $7(a)$ ). In our opinion, this is due to the low electron density at the end of the afterglow phase.

Note that the results of modelling enable only the qualitative analysis of argon/dust pulsed plasmas. There are some quantitative discrepancies between the results obtained from the model and the experimental data (figures 1 and 2). that can be attributed to some simplifications in the model.

In particular, the 0D model does not account for the plasma nonuniformity. Dusty plasmas are inhomogeneous and not isotropic, especially in the presence of self-organized structures, such as dust voids [2, 56]. Another difference between the model and the experiment is that the model describes the electron and metastable densities averaged over the entire plasma volume, while $n_{\mathrm{e}}$ and $n_{\mathrm{m}}$ in the experiment 
are the line-of-sight averaged densities measured at the midplane between the electrodes [27].

Further, to calculate the dust charge the ionization in the vicinity of a dust grain is neglected. If the ionization is important [57], the expression for the rate describing collection of ions by dust particles should be taken in the more complicate form $K_{i}^{d} \approx a_{d}^{2}\left(8 \pi e T_{i} / m_{i}\right)^{0.5}\left(1+\xi \tau+H \xi^{2} \tau^{2} \lambda_{S} n_{a} \sigma_{i a}(1+\kappa)\right)[57]$, where $\kappa=n_{a} K^{R 1} / v_{i n}, v_{i n}=\sqrt{e T_{i} / m_{i}} / \lambda_{\text {in }}$ and $\lambda_{\text {in }}=1 / n_{a} \sigma_{i a}$ is the ion mean-free path. Nevertheless, our calculations show that this effect does not change the dust charge and other plasma parameters significantly. This conclusion is not in contradiction with the results in [57]. In fact the results in [57] are obtained for the Maxwellian EEPF, while our results are calculated for the Druyvesteyn distribution. For the same $T_{\text {eff }}$ Druyvesteyn distribution has fewer electrons at high energies, which results in lower ionization rate. If we use the Maxwellian distribution (figure 5), $T_{\text {eff }} \leq 2.5 \mathrm{eV}$ and the ionization events also affect the dust charge insignificantly, what is in good agreement with the results on figure 1 in [57].

In our model, we assume that a small amount of acetylene may be present in the argon/dust plasma [27]. Due to the specific formation of the carbonacous dust particles in $\mathrm{Ar} / \mathrm{C}_{2} \mathrm{H}_{2}$ plasma the presence of small amount of impurities is inevitable [22, 27, 33]. However, the density of these impurities in the experiment is unknown at present. (To compare to the experiment the $\mathrm{C}_{2} \mathrm{H}_{2}$ density is assumed the same as in [27]). Therefore, to improve the model of the argon/dust pulsed plasma, one needs more experimental data on the plasma composition. Note also that the model does not consider the growth of dust particles. Therefore, the main task in the future will be to extend this model to describe the formation and growth of hydrocarbon grains in $\mathrm{Ar} / \mathrm{C}_{2} \mathrm{H}_{2}$ pulsed plasma.

To summarize, this study is only a first step on the way to self-consistent modelling of dusty pulsed plasmas with large dust densities. A complete approach should include the effects of the spatial nonuniformity and the ionization events on dust charge. We expect that including these effects in the model will give the time-dependencies for $n_{\mathrm{e}}$ and $n_{\mathrm{m}}$ which will be in better qualitative agreement with experimental data than those obtained using this 0D model. Nevertheless, the model considers the main processes occurring in an argon/dust pulsed plasma, and the results from the model are in a good qualitative agreement with available experimental data. The results on dusty pulsed plasmas here are relevant to many applications involving nonstationary plasmas containing impurities, especially gas discharge plasmas used for synthesis of novel nanomaterials.

\section{Acknowledgements}

$\mathrm{J} B$ and $\mathrm{E} \mathrm{K}$ would like to acknowledge the support from French Research Agency via ANR project PLASMABOND. I $\mathrm{S}$ is supported by Ministry of Education, Science and Technological Development of the Republic of Serbia according to the contract between the Institute of Technical Sciences and the Ministry No. 451-03-68/2020-14/200175.

\section{References}

[1] Bouchoule A (ed) 1999 Dusty Plasmas: Physics, Chemistry, and Technological Impacts in Plasma Processing (New York: Wiley)

[2] Vladimirov S V and Ostrikov K 2004 Phys. Rep. 393175

[3] Fortov V E, Ivlev A V, Khrapak S A, Khrapak A G and Morfill G E 2005 Phys. Rep. 4211

[4] Levchenko I, Keidar M, Cvelbar U, Mariotti D, Mai-Prochnow A, Fang J and Ostrikov K 2016 J. Phys. D: Appl. Phys. 49273001

[5] Kersten H, Deutsch H, Stoffels E, Stoffels W W, Kroesen G M W and Hippler R 2001 Contrib. Plasma Phys. 41598

[6] Winter J. 2000 Phys. Plasmas 73862

[7] Arp O, Block D and Piel A 2004 Phys. Rev. Lett. 93165004

[8] Mikikian M and Boufendi L 2004 Phys. Plasmas 113733

[9] Thomas H, Morfill G E, Demmel V, Goree J, Feuerbacher B and Möhlmann D 1994 Phys. Rev. Lett.73 652

[10] Le Picard R, Markosyan A H, Porter D H, Girshick S L and Kushner M J 2016 Plasma Chem. Plasma Process. 36941

[11] Denysenko I, Berndt J, Kovacevic E, Stefanovic I, Selenin V and Winter J 2006 Phys. of Plasmas 13073507

[12] Denysenko I B et al 2019 Plasma Phys. Control. Fusion 61 014014

[13] Denysenko I B et al 2020 J. Phys. D: Appl. Phys. 53135203

[14] Economou D J 2014 J. Phys. D: Appl. Phys. 47303001

[15] Samukawa S and Mieno T 1996 Plasma Sources Sci. Technol. 5 132

[16] Berndt J, Kovačević E, Stefanović I and Boufendi L $2009 \mathrm{~J}$. Appl. Phys. 106063309

[17] Hundt M, Sadler P, Levchenko I, Wolter M, Kersten H and Ostrikov K 2011 J. Appl. Phys.109 123305

[18] Bouchoule A, Plain A, Boufendi L, Blondeau J Ph and Laure C 1991 J. Appl. Phys. 701991

[19] Ivlev A et al 2003 Phys. Rev. Lett. 90055003

[20] Couëdel L, Samarian A A, Mikikian M and Boufendi L 2008 Phys. Plasmas 15063705

[21] Childs M A and Gallagher A 2000 J. Appl. Phys. 871086

[22] Denysenko I, Stefanović I, Sikimić B, Winter J, Azarenkov N A and Sadeghi N 2011 J. Phys. D: Appl. Phys. 44205204

[23] Denysenko I B, Stefanović I, Sikimić B, Winter J and Azarenkov N A 2013 Phys. Rev. E 88023104

[24] Berndt J, Kovacević E, Selenin V, Stefanović I and Winter J 2006 Plasma Sources Sci. Technol. 1518

[25] Stefanović I et al 2006 Phys. Rev. E 74026406

[26] Denysenko I B, Stefanović I, Azarenkov N A and Burmaka G P 2015 Phys. Plasmas 22023702

[27] Stefanović I, Sadeghi N, Winter J and Sikimić B 2017 Plasma Sources Sci. Technol. 26065014

[28] Kolobov V I and Godyak V A 1995 IEEE Trans. Plasma Sci. 23503

[29] Kortshagen U, Busch C and Tsendin L D 1996 Plasma Sources Sci. Technol. 51

[30] Kaganovich I D and Tsendin L D 1992 IEEE Trans. Plasma Sci. 2066

[31] Lieberman M A and Lichtenberg M A 2005 Principle of Plasma Discharges and Material Processing, 2 nd ed. (New Jersey: JohnWiley \& Sons Inc) 
[32] Ferreira C M, Loureiro J and Ricard A 1985 J. Appl. Phys. 57 82

[33] Stefanović I, Sadeghi N and Winter J 2010 J. Phys. D: Appl. Phys. 43152003

[34] Thorsteinsson E G and Gudmundsson J T 2009 Plasma Sources Sci. Technol. 18045001

[35] Godyak V A, Piejak R B and Alexandrovich B M 1992 Plasma Sources Sci. Technol. 136

[36] Phelps A V and Petrovic Z L 1999 Plasma Sources Sci. Technol. 8 R21

[37] Ali M A and Stone P M 2008 Int. J. Mass Spectrom. 27151

[38] Deutsch H et al 2004 Int. J. Mass Spectrom. 23339

[39] Kolokolov N B and Blagoev A B 1993 Phys.-Usp. 36152

[40] Lymberopoulos D P and Economou D J 1993 J. Appl. Phys. 73 3668

[41] Thorsteinsson E G and Gudmundsson J T 2010 J. Phys. D: Appl. Phys. 43115201

[42] Denysenko I B, Kersten H and Azarenkov N A 2015 Phys. Rev. E 92033102

[43] Maresca A, Orlov K and Kortshagen U 2002 Phys. Rev. E 65 056405

[44] Mityureva A A and Smirnov V V 2004 Opt. Spektrosk. 97508 [45] Denysenko I, Yu M Y, Ostrikov K and Smolyakov A 2004 Phys. Rev. E 70046403

[46] Yanguas-Gil A, Cotrino J and Alves L 2005 J. Phys. D: Appl. Phys. 381588

[47] Bogaerts A, Gijbels R and Vlèek J 1998 J. Appl. Phys. 84121

[48] Khrapak S A et al 2005 Phys. Rev. E 72016406

[49] Byrne G D and Thompson S 2013 VODE_F90 Support Page, http: //www.radford.edu/ thompson/vodef90web

[50] Sadeghi N 2004 J. Plasma Fusion Res. 80767

[51] Sikimić B, Stefanović I, Denysenko I B and Winter J 2013 Plasma Sources Sci. Technol. 22045009

[52] Despiau-Pujo E, Brihoum M, Bodart P, Darnon M and Cunge G 2014 J. Phys. D. Appl. Phys. 47455201

[53] Lieberman M A and Ashida S 1996 Plasma Sources Sci. Technol. 5145

[54] Carbone E, Sadeghi N, Vos E, Hübner S, van Veldhuizen E, van Dijk J, Nijdam S and Kroesen G 2015 Plasma Sources Sci. Technol. 24015015

[55] Gudmundsson J T 2001 Plasma Sources Sci. Technol. 1076

[56] Mikikian M et al 2003 New J. Phys. 519

[57] Khrapak S A and Morfill G E 2012 Phys. Plasmas 19024510 